\title{
Systems with Fractional Variable-Order Difference Operator of Convolution Type and Its Stability
}

\author{
Dorota Mozyrska, Malgorzata Wyrwas \\ Faculty of Computer Science, Bialystok University of Technology, \\ Wiejska 45a, 15-351 Bialystok, Poland \\ d.mozyrska@pb.edu.pl
}

\begin{abstract}
In the paper the Grünwald-Letnikov-type linear fractional variable order discrete-time systems are studied. The conditions for stability and instability are formulated. The regions of the systems stability are determined accordingly to locus of eigenvalues of a matrix associated to the considered system.
\end{abstract}

Index Terms-Asymptotic stability; Fractional calculus; Stability analysis; System analysis and design.

\section{INTRODUCTION AND PRELIMINARIES}

Recently, theory of fractional dynamical systems has become one of the essential tool for modelling in various technical disciplines, particularly in electrotechnics, chemistry, electrochemistry or viscoelasticity, see for instance [1]-[3]. The systems with the fractional variable orders for continuous-time as well for discrete-time have been studied in [4]-[8]. In the paper we deal with discretetime systems described by the Grünwald-Letnikov operator of convolution type, and with step $h>0$. This allow us to introduce the continuous case by using the limit with $h$ tending to zero. We formulate and prove the conditions for the stability of the considered discrete-time systems. We determined the regions of location of eigenvalues of matrices associated to the systems in order to guarantee the asymptotic stability of the considered systems. These regions were presented as the images of some map associated with the GL-VFOBD- $h$. Some of the frames of these regions are illustrated in the example.

Definition 1 ([4]). For $k, l \in \mathbb{Z}$ and a given order function $v(\cdot)$ we define the oblivion function, as a discrete function of two variables, by its values $a^{[v(l)]}(k)$ as follows

$$
a^{[v(l)]}(k):=(-1)^{k} \frac{v(l)[v(l)-1] \cdots[v(l)-k+1]}{k !},
$$

where $k>1, a^{[v(l)]}(0)=0, a^{[v(l)]}(1)=1$.

Formula (1) in Definition 1 can be equivalently expressed by the following recurrence with respect to $k \in \mathbb{N}$ :

Manuscript received 4 January, 2018; accepted 7 April, 2018.

The work was supported by Polish funds of National Science Center, granted on the basis of decision DEC-2016/23/B/ST7/03686.

$$
\begin{cases}a^{[v(l)]} & (0):=1, \\ a^{[v(l)]} & (k):=a^{[v(l)]}(k-1)\left[1-\frac{v(l)+1}{k}\right] \text { for } k \geq 1,\end{cases}
$$

where $(h \mathbb{N})_{0}:=\{0, h, 2 h, \ldots\},, h>0$

Definition 2. Let $h>0$ and $x:(h \mathbb{N})_{0} \rightarrow \mathbb{R}$ be a bounded function. The Grünwald-Letnikov variable-, fractional-order backward difference with step $h>0$ (GL-VFOBD-h) of function $x(\cdot)$ with an order function $v: \mathbb{Z} \rightarrow \mathbb{R}_{+} \cup\{0\}$ started at $k_{0}=0$ is defined as a finite sum

$$
\left(\Delta_{h}^{[v(\cdot)]} x\right)(k h):=\sum_{i=0}^{k} h^{-v(i)} a^{[v(i)]}(i) x(k h-i h) .
$$

Definition 3. Let $x: \mathbb{R}_{+} \cup\{0\} \rightarrow \mathbb{R}$ be a continuous bounded real valued function. The Grünwald-Letnikov variable-, fractional-order differential operator of function with an order function $v: \mathbb{Z} \rightarrow \mathbb{R}_{+} \cup\{0\}$ started at $t_{0}=0$ is defined as

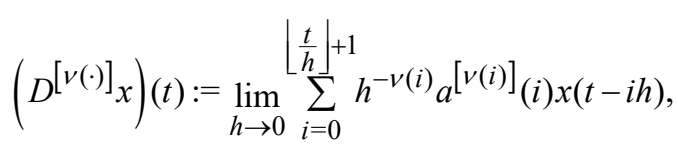

where $h>0, t \geq 0$. The sign \lfloor\rfloor denotes the floor function.

\section{DifFERENCE Systems With Fractional VARIABLE ORDER}

Let us consider the system with a variable-order the following form

$$
\left(\Delta_{h}^{[v(\cdot)]} x\right)(k h)=A x(k h-h)+B u(k h),
$$

where $k \geq 1$, with initial condition $x(0)=x_{0} \in \mathbb{R}^{n}$, where function $u: \mathbb{N}_{0} \rightarrow \mathbb{R}^{m}$ is an input function, $A \in \mathbb{R}^{n \times n}$, $B \in \mathbb{R}^{n \times m}$ 
By (3) system (5) can be rewritten in the following recurrence way

$$
\begin{gathered}
x(k h)=-\sum_{i=1}^{k} h^{v(0)-v(i)} a^{[v(i)]}(i) x(k h-i h)+ \\
+h^{v(0)} A x(k h-h)+h^{v(0)} B u(k h),
\end{gathered}
$$

where $k \geq 1$.

Proposition 4. System (5) with initial value $x(0) \in \mathbb{R}^{n}$ has the unique solution given by

$x(k h)=\Phi(k)(x(0)-B u(0))+B \sum_{i=0}^{k-1} \Phi(k-1-i) u(i h+h)$,

where

$$
\Phi(k)=Z^{-1}\left[\left(\alpha(z) I-A z^{-1}\right)^{-1}\right](k)
$$

where $\alpha(z)$ is defined by

$$
\alpha(z):=Z[a](z)=\sum_{i=0}^{\infty} h^{-v(i)}(-1)^{i}\left(\begin{array}{c}
v(i) \\
i
\end{array}\right) z^{-i}
$$

Proof. Note that (5) can be equivalently rewritten as

$$
\left(\Delta^{[v(\cdot)]} x\right)(k h+h)=A x(k h)+B u(k h+h),
$$

where $k \geq 0$. Then taking the one-side Z-transform of system (10), see more in [9], one gets the following equation in Z-domain

$$
z \alpha(z) X(z)-z x(0)=A X(z)+B z U(z)-B z u(0),
$$

where $\alpha(z)=Z[a](z), X(z)=Z[\bar{x}](z), \quad \bar{x}(k):=x(k h)$ and $U(z)=Z[\bar{u}](z), \bar{u}(k):=u(k h)$. Consequently

$$
\begin{aligned}
X(z)= & \left(\alpha(z) I-z^{-1} A\right)^{-1}(x(0)-B u(0))+ \\
& +B\left(\alpha(z) I-z^{-1} A\right)^{-1} U(z) .
\end{aligned}
$$

In order to get the thesis one needs to take inverse Ztransform of (12).

First let us formulate a condition for instability of system (5).

Proposition 5. Let $\operatorname{spec}(A)=\left\{\lambda_{\ell}: \ell=1, \ldots, k\right\}, k \leq n$. If there is $\lambda \in \operatorname{spec}(A)$ such that

$$
|\lambda|>\left(a_{\lambda}^{2}+b_{\lambda}^{2}\right)^{0.5}
$$

where

$$
\begin{aligned}
& a_{\lambda}=\sum_{k=0}^{\infty} h^{-v(k)} a^{[v(k)]}(k) \cos \left((k-1) \phi_{\lambda}\right), \\
& b_{\lambda}=\sum_{k=0}^{\infty} h^{-v(k)} a^{[v(k)]}(k) \sin \left((k-1) \phi_{\lambda}\right),
\end{aligned}
$$

and $\phi_{\lambda}=\arg (\lambda)$, then system (5) is unstable.

Proof. Let $\alpha$ be given by (9) and $\phi_{\lambda} \in \mathbb{R}$. Since $(-1)^{k}\left(\begin{array}{c}v(k) \\ k\end{array}\right)=a^{[v(k)]}(k)$ and

$$
\begin{gathered}
\sum_{k=0}^{\infty} h^{-v(k)} a^{[v(k)]}(k) e^{-(k-1) i \phi_{\lambda}}= \\
=e^{i \phi_{\lambda}} \sum_{k=0}^{\infty} h^{-v(k)} a^{[v(k)]}(k) e^{-k i \phi_{\lambda}}= \\
=e^{i \phi_{\lambda}} A\left(e^{i \phi_{\lambda}}\right),
\end{gathered}
$$

one gets

$$
\left(a_{\lambda}^{2}+b_{\lambda}^{2}\right)^{0.5}=\left|e^{i \phi_{\lambda}} \alpha\left(e^{i \phi_{\lambda}}\right)\right|=\left|\alpha\left(e^{i \phi_{\lambda}}\right)\right|
$$

Then by (13) we get that $\lambda$ does not belong to the set $\{z \alpha(z):|z| \leq 1\}$. Hence the eigenvalue $\lambda$ of the matrix $A$ lies outside of the image of unit circle in the mapping $\bar{\alpha}: \mathbb{C} \rightarrow \mathbb{C}$ given by $\bar{\alpha}(z)=z \alpha(z)$. Consequently, system (5) is unstable.

Since values of coefficients $a^{[v(k)]}(k)$ are tending very fast to zero, one can approximate series in condition (8) by taking the right hand side for some steps. Observe that for $s>0$ one gets

$$
\begin{gathered}
\left(a_{\lambda}^{2}+b_{\lambda}^{2}\right)^{0.5} \geq \\
\geq\left(\left(\sum_{k=0}^{s} h^{-v(k)} a^{[v(k)]}(k) \cos \left((k-1) \phi_{\lambda}\right)\right)^{2}+\right. \\
\left.+\left(\sum_{k=0}^{S} h^{-v(k)} a^{[v(k)]}(k) \sin \left((k-1) \phi_{\lambda}\right)\right)^{2}\right)^{0.5} .
\end{gathered}
$$

In practice it is enough to calculate the right side of (13) for some steps.

Now let us give the necessary and sufficient condition for the stability of system (5).

Proposition 6. Let $\operatorname{spec}(A)=\left\{\lambda_{i}: i=1, \ldots, k\right\}, k \leq n$. If for all $i=1, \ldots, k$ we have that

$$
\lambda_{i} \in\{z \alpha(z):|z|<1\}
$$

then system (5) is asymptotically stable.

Proof. Observe that if all roots of the equation $\operatorname{det}(z \alpha(z) I-A)=0$ are inside the unit circle, then system (5) (or equivalently, (6)) is asymptotically stable, see for instance [9]. Note that $A=P J P^{-1}$, where $P$ is invertible and $J=\operatorname{diag}\left(J_{1}, \ldots, J_{s}\right)$ and $J_{l}$ are Jordan's blocks of order $r_{l}, l=1, \ldots, s$. Since

$$
\begin{gathered}
\operatorname{det}(z \alpha(z) I-A)=\operatorname{det}\left(z \alpha(z) I-P J P^{-1}\right)= \\
=\operatorname{det}(z \alpha(z) I-J),
\end{gathered}
$$


all roots of the equation $\operatorname{det}(z \alpha(z) I-A)=0$ are inside the unit circle if and only if all eigenvalues of $A$ belong to $\{z \alpha(z):|z|<1\}$.

Remark 7. Observe that the set $\left\{e^{i \varphi} \alpha\left(e^{i \varphi}\right): \varphi \in[0,2 \pi)\right\}$ is the frame of the set $\{z \alpha(z):|z|<1\}$. Then the fact that all eigenvalues of $A$ are inside the set restricted by the figure $\left\{e^{i \varphi} \alpha\left(e^{i \varphi}\right): \varphi \in[0,2 \pi)\right\} \quad$ guarantees system (5) (or equivalently, (6) or (10)) to be asymptotically stable.

Example 8 . We consider in the example the system with three different order functions. The considered system has

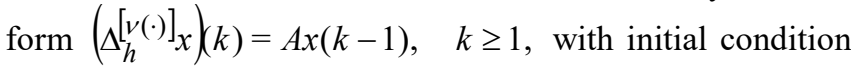
$x(0)=x_{0} \in \mathbb{R}^{2}$ and matrix

$$
A=\frac{1}{2 h}\left[\begin{array}{rr}
1 & -1 \\
1 & 1
\end{array}\right] \text {. }
$$

Then

$$
\operatorname{spec}(A)=\left\{\frac{1}{2 h} \pm \frac{1}{2 h} i\right\} .
$$

We present for systems and their orders the stability regions. In the same figures we illustrate by crosses eigenvalues of $A$.

- In Fig. 1 and Fig. 2 we present the frames of regions of stability for order functions given by $v_{1}(k)=1-e^{-a k}$, $a \in\{0.1,0.3,0.6\}$. Order functions $v_{1}(\cdot)$ are increasing functions with values from $[0,1]$. In considered situations we receive asymptotically stable system.

- In Fig. 3 and Fig. 4 we present the frames of regions of stability for order functions given by $v_{2}(k)=e^{-a k}$, $a \in\{0.1,0.3,0.6\}$. Order functions $v_{2}(\cdot)$ are increasing functions with values from $[0,1]$. Then, we have stability only for $a=0.6$ if $h=0.1$, see Fig. 3 and for $a \in\{0.3,0.6\}$ in the case when $h=0.01$, see Fig. 4 .

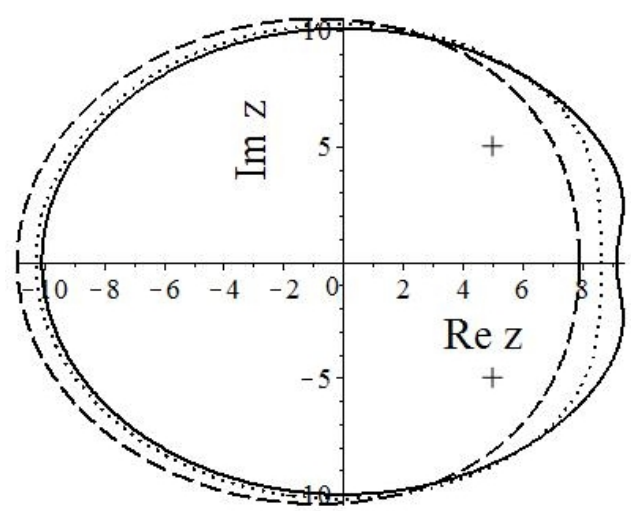

$$
\mathrm{a}=0.1 \cdots \cdots \mathrm{a}=0.3--\mathrm{a}=0.6
$$

Fig. 1. Stability regions for system (5) with order function $v_{1}(k)=1-e^{-a k}$, for $a \in\{0.1,0.3,0.6\}, h=0.1$. Crosses denotes locus of eigenvalues of matrix $A$.

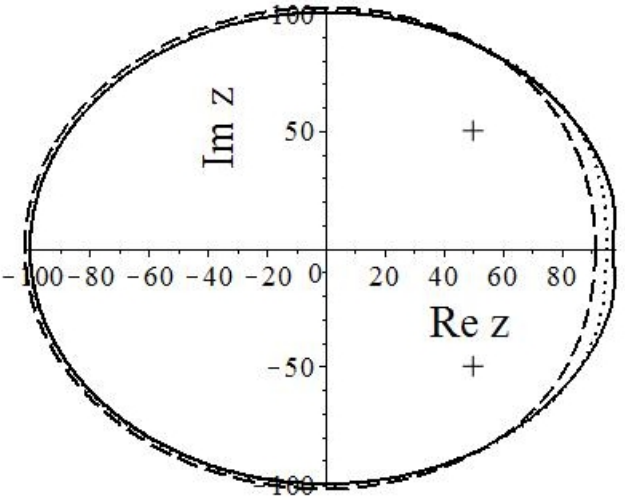

$$
-\mathrm{a}=0.1 \cdots \cdots \mathrm{a}=0.3--\mathrm{a}=0.6
$$

Fig. 2. Stability regions for system (5) with order function $v_{1}(k)=1-e^{-a k}, \quad$ for $\quad a \in\{0.1,0.3,0.6\}, \quad h=0.01$. Crosses denotes locus of eigenvalues of matrix $A$.

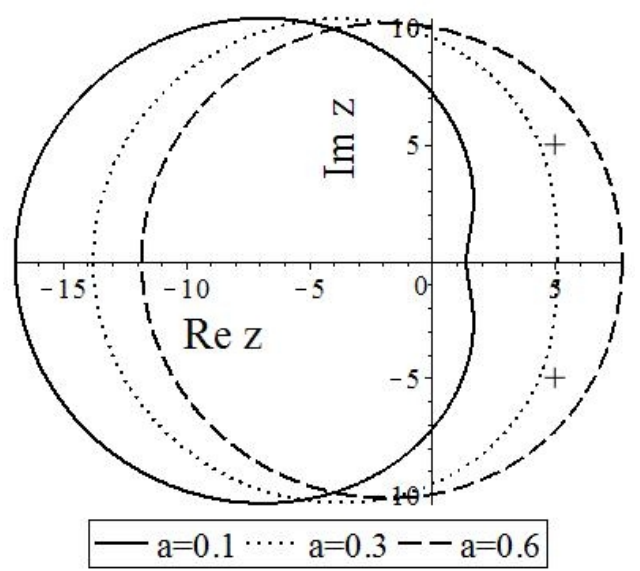

Fig. 3. Stability regions for system (5) with order function $v_{2}(k)=e^{-a k}$, for $a \in\{0.1,0.3,0.6\}, h=0.1$. Crosses denotes locus of eigenvalues of matrix $A$.

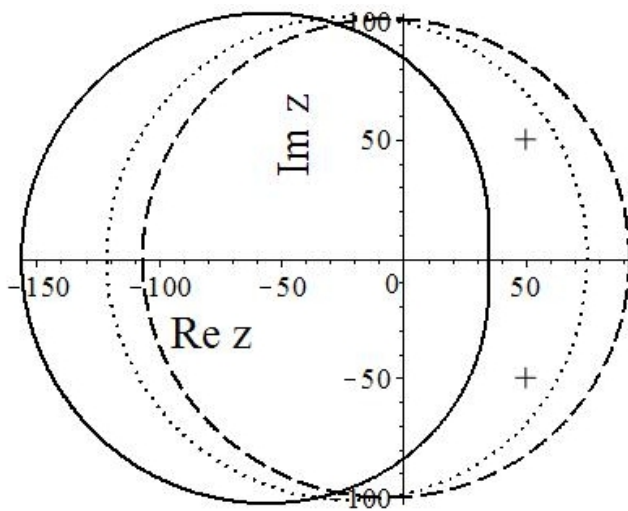

$$
\mathrm{a}=0.1 \cdots \cdots \mathrm{a}=0.3--\mathrm{a}=0.6
$$

Fig. 4. Stability regions for system (5) with order function $v_{2}(k)=e^{-a k}$, for $a \in\{0.1,0.3,0.6\}, h=0.01$. Crosses denotes locus of eigenvalues of matrix $A$.

- In Fig. 5 and Fig. 6 we present the frames of regions of stability for order functions given by $v_{3}(k)=|\sin (a k)|$, $a \in\{1,3,6\}$ and two steps $h=0.1$ and $h=0.01$. In both cases of $h$ we do not have stability for $a=1$. 


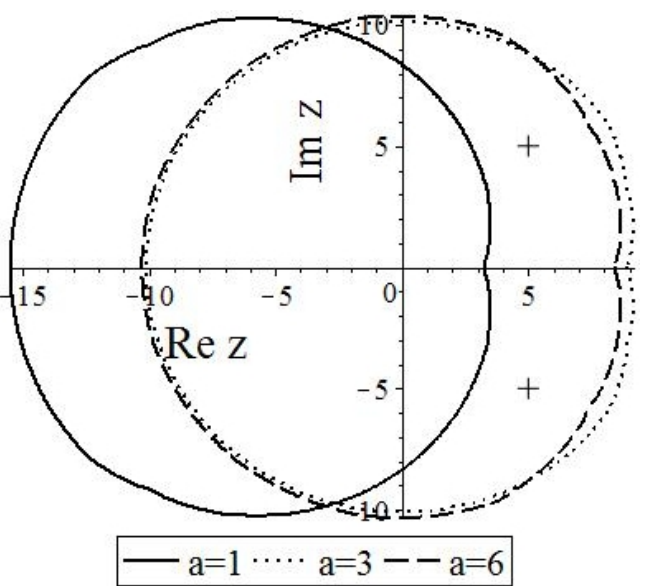

Fig. 5. Stability regions for system (5) with order function $v_{3}(k)=|\sin (a k)|$, for $a \in\{1,3,6\}$ and $h=0.1$. Crosses denotes locus of eigenvalues of matrix.

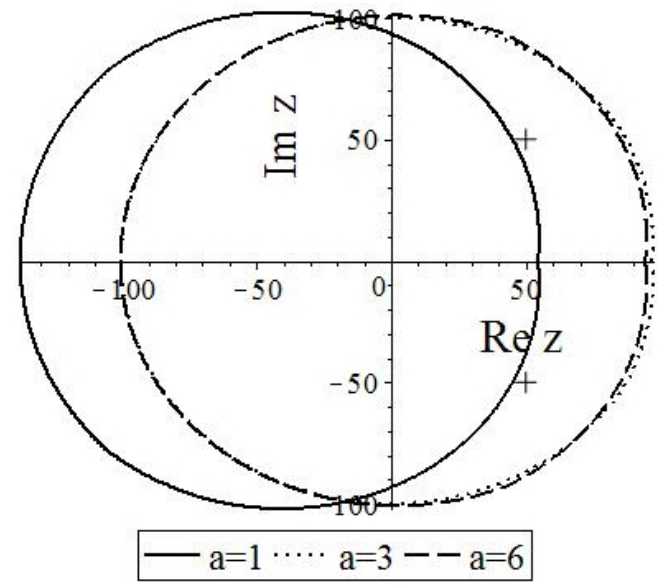

Fig. 6. Stability regions for system (5) with order function $v_{3}(k)=|\sin (a k)|$, for $a \in\{1,3,6\}$ and $h=0.01$. Crosses denotes locus of eigenvalues of matrix.

\section{FRACTIONAL-, VARIABLE-ORDER EQUATIONS}

In this section we will study the stability of equation. Let us take $A=\lambda \in \mathbb{R}$. Then $\operatorname{spec}(A)=\{\lambda\}$. Let us consider such equation with a variable-order as follows

$$
\left(\Delta_{h}^{[v(\cdot)]} x\right)(k h)=\lambda x(k h-h)+b u(k h)
$$

where $k \geq 1$, with initial condition $x(0)=x_{0} \in \mathbb{R}$. Then, (9) can be rewritten in the following recurrence way

$$
\begin{gathered}
x(k h)=-\sum_{i=1}^{k} h^{v(0)-v(i)} a^{[v(i)]}(i) x(k h-i h)+ \\
+h^{v(0)} \lambda x(k h-h)+h^{v(0)} b u(k h),
\end{gathered}
$$

where $k \geq 1$ and $x(0)=x_{0} \in \mathbb{R}$ is given.

From Proposition 4 for $A=\lambda, B=b$ and $I=1$ one gets the following result:

Proposition 9. Equation (23) with initial value $x(0) \in \mathbb{R}$ has the unique solution given by

$$
x(k h)=\Phi(k)(x(0)-b u(0))+b \sum_{i=0}^{k-1} \Phi(k-1-i) u(i h+h)
$$

where

$$
\Phi(k)=Z^{-1}\left[\left(\alpha(z) I-\lambda z^{-1}\right)^{-1}\right](k),
$$

where $\alpha(z)$ is defined by (9).

The sufficient condition for the asymptotic stability of equation (23) is as follows:

Proposition 10. If

$$
-\sum_{i=0}^{\infty} h^{-v(i)}\left(\begin{array}{c}
v(i) \\
i
\end{array}\right)<\lambda<\sum_{i=0}^{\infty} h^{-v(i)}(-1)^{i}\left(\begin{array}{c}
v(i) \\
i
\end{array}\right),
$$

then (23) is asymptotically stable.

Proof. We are interested in the set of roots of the equation $\alpha(z)-\lambda z^{-1}=0$. Then $\lambda=z \alpha(z)$. Observe that for the considered scalar equation the points $z=-1$ and $z=1$ correspond to the border of the asymptotic stability of the considered system. Hence, if

$$
\lambda<\sum_{i=0}^{\infty} h^{-v(i)}(-1)^{i}\left(\begin{array}{c}
v(i) \\
i
\end{array}\right)
$$

for $z=1$ and for $z>-1$ one gets

$$
-\lambda<\sum_{i=0}^{\infty} h^{-v(i)}\left(\begin{array}{c}
v(i) \\
i
\end{array}\right)
$$

Therefore (27) guarantees $\lambda \in\{z \alpha(z):|z|<1\}$ and consequently, (23) is asymptotically stable.

Using the properties of order function and Proposition 10 one gets the following result:

Corollary 11. If

$$
|\lambda|<\sum_{i=0}^{\infty} h^{-v(i)}\left(\begin{array}{c}
v(i) \\
i
\end{array}\right),
$$

then (23) with $\lambda<0$ and the increasing order function $v(\cdot)$ is asymptotically stable.

\section{CONCLUSiOnS}

In the paper the stability of the Grünwald-Letnikov-type linear fractional-, variable-order discrete-time systems with step $h>0$ was studied. We investigate systems described by the Grünwald-Letnikov operator of convolution type, and with step $h>0$. The convolution-type operator allow us to use Z-transform and formulate and prove the conditions for the stability of the considered discrete-time systems. We determined the regions of location of eigenvalues of matrices associated to the systems in order to guarantee the asymptotic stability of the considered systems. Summarizing we have produced conditions and descriptions for the stability of the Grünwald-Letnikov-type linear fractional-, variable-order discrete-time systems with step $h>0$.

\section{REFERENCES}

[1] G. R. J. Cooper, D. R. Cowan, "Filtering using variable order vertical derivatives", Computers \& Geosciences, vol. 30, no. 5, pp. 455-459, 2004. DOI: $10.1016 /$ j.cageo.2004.03.001

[2] P. Ostalczyk, "Discrete fractional calculus. Applications in control 
and image processing”, Series in Computer Vision, vol. 4, 2016. DOI: $10.1142 / 9833$

[3] D. Sierociuk, A. Dzielinski, "Stability of discrete fractional order state-space systems", J. Vibration and Control, vol. 14, no. 9-10 pp. 1543-1556, 2008. DOI: 10.1177/1077546307087431.

[4] D. Mozyrska, P. Ostalczyk, "Variable-fractional-order GrünwaldLetnikov backward difference selected properties", in Proc. 39th Int Conf. Telecommunications and Signal Processing, 2016. DOI: 10.1109/TSP.2016.7760959.

[5] D. Mozyrska, P. Ostalczyk, "Generalized fractional-order discretetime integrator", Complexity, 2017, p. 11. DOI: $10.1155 / 2017 / 3452409$

[6] P. Ostalczyk, "Stability analysis of a discrete-time system with a variable-, fractional-order controller", Bulletin of The Polish Academy of Sciences, Technical Science, vol. 58, no. 4, pp. 613-619, 2010 DOI: 10.2478/v10175-010-0063-x.

[7] D. Sierociuk, W. Malesza, "Fractional variable order discrete-time systems, their solutions and properties", International Journal of Systems Science, vol. 48, no. 14, pp. 3098-3105, 2017. DOI: 10.1080/00207721.2017.1365969.

[8] D. Valerio, J. Sada Costa, "Variable-order fractional derivatives and their numerical approximations", Signal Processing, vol. 91, no. 3, pp. 470-483, 2011. DOI: 10.1016/j.sigpro.2010.04.006.

[9] D. Mozyrska, M. Wyrwas, "The Z-transform method and delta type fractional difference operators", Discrete Dynamics in Nature and Society, 2015, p. 12. DOI: 10.1155/2015/852734. 